\title{
IDENTIDADE ORGANIZACIONAL
}

\author{
Miguel P. Caldas \\ Mestre e Doutorando em Administração de Empresas na \\ EAESP/FGV. Professor do Departamento de Administração \\ Geral e Recursos Humanos da EAESP/FGV e consultor de \\ empresas. \\ E-mail: mcaldas@eaesp.fgvsp.br \\ Thomaz Wood Jr. \\ Mestre e Doutorando em Administração de Empresas na \\ EAESP/FGV. Professor do Departamento de Produção, \\ Logística e de Operações Industriais da EAESP/FGV e \\ consultor de empresas. \\ E-mail: twood@eaesp.fgvsp.br
}

RESUMO: Neste artigo teórico, os autores revisitam o conceito de identidade, advogando o melhor entendimento de sua complexidade e sua utilização mais abrangente na análise organizacional. Enquanto exploram as origens do conceito e os caminhos de sua evolução por vários campos do conhecimento, os autores desenvolvem um quadro conceitual de referência, que diferencia as muitas abordagens existentes sobre identidade, a partir de duas dimensões básicas: dimensão do objeto focal e dimensão da observação. A primeira distingue o objeto sobre $o$ qual o conceito é aplicado (ex.: indivíduo, grupo, organização etc.); a segunda distingue as formas pelas quais a identidade é observada (do interiormente observado - self - até o externamente observado - imagem). A sobreposição dessas duas dimensões define diversos quadrantes, onde se podem distinguir as diferentes abordagens existentes sobre identidade. Enfim, o modelo é expandido com uma terceira dimensão (da definição de identidade), que põe em debate os alicerces do próprio conceito: a idéia de algo central, distintivo e duradouro.

ABSTRACT: In this theoretical article, the authors revisit the concept of identity, proposing the better understanding of its complexity, as well as its broader use in organizational analysis. While exploring the origins of the concept and the trends of its evolution through several fields of knowledge, the authors develop a conceptual framework, which differentiates the many existing perspectives on identity based on two key dimensions: the focal object dimension and the observation dimension. The former distinguishes the object to which the concept is applied (e.g., an individual, a group, an organization etc.); the latter differentiates how identity is observed (from the innerly observed - self - to the externally observed - image). The combination of these two dimensions defines several quadrants, in which it is possible to portrait the different existing perspectives on identity. At last, the model is expanded with a third dimension (the identity definition), which debates the basic foundations of the concept: of this being something central, distinctive and enduring.

PALAVRAS-CHAVE: identidade, identidade organizacional, mudança organizacional, self, auto-conceito, organização virtual.

KEY WORDS: identity, organizational identity, organizational change, self, self-concept, virtual organization. 
A RJ\&M Autopeças (nome fictício) é uma empresa brasileira do setor automobilístico. Fundada há quarenta anos, conserva muitas características de empresa tradicional e familiar: processo decisório centralizado, estrutura hierarquizada $\mathrm{e}$ forte presença dos donos. Com as mudanças ocorridas na economia e no setor automobilístico no início dos anos noventa, a RJ\&M passou a sentir sua posição competitiva amieaçada. Em 1994, a RJ\&M contratou uma empresa de consultoria para mudar seu modelo de negócios e implementar um processo mais moderno de gestão. $O$ projeto de mudança foi dividido em três fases: diagnóstico estratégico e organizacional, redesenho do modelo organizacional e implantação.

Vejamos três cenas ocorridas durante o processo de mudança:

- Cena 1 - durante o diagnóstico. Em uma reunião com proprietários e diretores da empresa, a equipe de consultoria apresenta os resultados de uma pesquisa de opinião, realizada $\mathrm{ccm}$ clientes e fornecedores da RJ\&M. Para surpresa da maioria dos presentes, que viam sua empresa como modelo de sucesso, os resultados são negativos. Muitos clientes reclamam do atendimento e fornecedores vêem a RJ\&M como pouco eficiente e desatenta a oportunidades de melhoria. Como um todo, o diagnóstico é dissonante da visão dominante e gera um sentimento de desagrado nas pessoas. No entanto, depois do mal-estar inicial, o diagnóstico ajuda os executivos a alterarem sua percepção sobre a empresa, reforçando o consenso em torno da necessidade de mudar.

- Cena 2 - durante o redesenho. São realizadas reuniões individuais com os gerentes. O objetivo é desenvolver a nova estrutura organizacional. Visando sistematizar o trabalho, cada gerente é estimulado a identificar seus clientes internos e externos, os produtos e serviços fornecidos a esses clientes e suas expectativas em relação a esses produtos e serviços. Sugere-se também que descrevam qual a razão de ser de sua área, na forma de uma declaração de missão. Para surpresa dos consultores, esta última é a parte mais difícil do trabalho e poucos gerentes conseguem descrever algo supostamente simples e objetivo quanto à razão da existência da sua área para a organização. A solução encontrada é marcar reuniões específicas para discutir o tema e ajudar os gerentes a melhor compreenderem seu novo papel. Só assim é possível dar continuidade ao desenvolvimento da nova estrutura organizacional.

- Cena 3 - durante a implantação. Na fase de implantação, cada gerente assume o papel de condutor do processo de mudança na sua área. Entretanto, dois deles começam a mostrar sinais de estresse diante da dificuldade de adaptar-se à nova realidade. Embora declarem compreender seu novo papel, não se vêem em condições de exercê-lo. As justificativas são variadas: falta de tempo para exercer novas funções, capacitação insuficiente, perfil inadequado etc.

Um ponto comum nessas três cenas é a presença da questão da identidade.

Na primeira cena, a identidade organizacional aparece em diversas dimensões: primeiro, surge na forma pela qual a organização é percebida pelo meio, na sua imagem para clientes e fornecedores. Surge novamente permeando a forma pela qual a organização, representada por seus executivos, percebe a si mesma - autopercepção. Fica aí clara a interação entre diversas dimensões, compondo um ciclo de mudança: o "como sou visto" ajudando a modificar o "como me vejo". Este último, em um segundo momento, gerando mudanças que vão alterar o "como sou" que, finalmente, deve alterar o "como sou visto", completando um ciclo.

Na segunda cena, é a identidade do grupo que aparece, expressa pela dificuldade dos gerentes em redefinir seu papel e o dos seus liderados no novo modelo organizacional. Fica evidente para os consultores a ruptura que as mudanças ambientais e o processo de mudança organizacional trazem para esse grupo. Como a discussão que se segue aborda esses temas, explorando a questão da identidade, consegue-se rearticular o processo. $O$ trabalho resulta em profundo questionamento quanto à razão de ser de cada um e gera novas identidades organizacionais para muitos gerentes $\mathrm{e}$ muitas áreas.

Na terceira cena, o que se percebe é uma interação conflituosa entre a nova identidade da função ou da área, definida na etapa anterior (de redesenho organizacional), e a identidade individual. Os dois gerentes mencionados não conseguem reposicionar-se pessoal- 
1. Ver NKOMO, S. \& COX, Jr. T. Diverse identities in organizations. In: CLEGG, S. , HARDY C. \& NORD, W. (Orgs), Handbook of Orga nization Studies. London: Sage, 1996, p. 338 356.

2. Em um trabalho recente, um framework inicial nesse sentido fol proposto por Wood Caldas para analisar processos radicais de $\mathrm{mu}$ dança: ver WOOD Jr., T., CALDAS, M.P. Quem tem medo de eletrochoque? Identidade, ternpias convulsivas e mudança organizacional. RAE-Revista de Administracão de Empresas, 35 5 de Administração de Empresas, v. modelo mais abrangente.

3. Ver RODRIGUES, S. B. Corporate culture and identity: De-institutionalization in a Brazilian telecommunications company. Anais do 17른 vador, $B A, 1993$

4. ver COPI, I. M. \& COHEN, C. Introduction to logic (8th ed.). New York: Macmillan, 1990

5. Ver WHITEHEAD, A. N. \& RUSSELL, B. Principia Mathematica (2nd ed.) Cambridge: Cambridge University Press, 1927.

6. Ver KAHN, C. H. The art and thought of Heraclitus: An edition of the fragments with translation and commentary. Cambridge: Cambridge University Press, 1979. mente para o novo papel que o modelo organizacional thes confere. O resultado aparece na forma de estresse e resistência à mudança.

Essas três cenas ilustram algumas das muitas possibilidades de utilização do conceito de identidade na análise organizacional. Também permitem perceber como as várias formas de identidade articulam-se. A imagem externa da organização, alterando sua auto-imagem e originando ou catalisando mudanças. Essas mudanças alterando e afetando a percepção dos grupos, gerando dificuldades e frustrações em âmbito funcional e individual e gerando outras mudanças. Uma dinâmica complexa, caracterizada por ações simultâneas dos vários atores organizacionais, definindo e redefinindo continuamente percepções e realidades.

Situações como essas, onde atores organizacionais - sejam indivíduos, grupos, ou a própria organização - questionam profundamente $o$ que são, acontecem todos os dias. Processos organizacionais - como fusões, aquisições e reestruturações — são eventos estreitamente ligados a questionamentos dessa natureza. Em contextos de mudanças amplas e complexas, como o da RJ\&M, transformações no nível macro provocam questionamentos e reconceituações em cadeia, do nível organizacional ao individual. Uma vez que todas essas situações parecem tornar-se cada dia mais comuns nas organizações, a idéia de identidade organizacional torna-se um conceito valioso.

Por outro lado, a utilização da noção de identidade não é simples. A maior dificuldade que se apresenta ao pesquisador é justamente sua complexidade e amplitude de sentidos. Estes variam nos diversos campos teóricos que empregam o termo e mesmo entre correntes dentro de cada um desses campos. Além disso, são raros os trabalhos que se preocupam em desenvolver tipologias ${ }^{1}$ e quadros de referência. ${ }^{2}$ No Brasil, poucos sequer têm abordado o tema. ${ }^{3}$ Nosso propósito neste trabalho é preencher esse vazio. Para tanto, revisaremos diversas abordagens baseadas na idéia de identidade, com ênfase no conceito de identidade organizacional. Acreditamos que nossa principal contribuição é o desenvolvimento de um quadro conceitual que, esperamos, torne tratável o conceito de identidade no estudo de fenômenos organizacionais.

O restante do artigo está estruturado da seguinte forma:
- na próxima seção, abordaremos as origens do conceito de identidade;

- em seguida, na terceira seção, proporemos um primeiro quadro conceitual para mapear as abordagens existentes sobre identidade, desenvolvido a partir de duas dimensões fundamentais: a dimensão do objeto focal (a quem o conceito é aplicado) e a dimensão da observação (a perspectiva a partir da qual o conceito é aplicado);

- na quarta seção, ampliaremos o quadro conceitual, dotando-o de uma terceira dimensão, a qual denominamos dimensão $d a$ definição de identidade. Tal dimensão confere profundidade ao quadro, relacionando-se a algumas discussões contemporâneas fundamentais sobre a questão de identidade; $\mathrm{e}$

- na seção final realizaremos um sumário do trabalho e procuraremos indicar direções para futuras pesquisas.

\section{ORIGENS DO CONCEITO DE IDENTIDADE}

O uso popular dos conceitos de identidade e de self tem fortes raízes no pensamento clássico. O emprego original do termo identidade como uma propriedade é tão antigo quanto a lógica, a álgebra, e a filosofia. ${ }^{4} \mathrm{O}$ "princípio da identidade", por exemplo, é um dos axiomas da lógica. Segundo ele, dada uma entidade, ela é idêntica a si mesma; ou seja, para qualquer $x, x$ é sempre igual a $x$. $\mathrm{Na}$ álgebra, diz-se existir identidade quando duas expressões são representadas pelo mesmo número. ${ }^{5} \mathrm{O}$ uso popular do termo relaciona-se também à filosofia clássica, que traz a noção de identidade associada à idéia de permanência, singularidade e unicidade do que constitui a realidade das coisas. Tal conceito é atribuído a Heráclito, que viveu entre os séculos VI e V a.C. É seu o famoso argumento de que um indivíduo pode, e ao mesmo tempo não pode, entrar duas vezes no mesmo rio, porque apesar de todos os seus constituintes materiais terem sofrido uma transformação, de certa forma o rio ainda é o mesmo. $O$ interesse de Heráclito pela idéia de unicidade gerou um conceito de identidade que influenciou centenas de gerações depois dele. ${ }^{6}$ Dessas duas origens principais - lógica e filosofia clássicas - a idéia de identidade tornou-se universal, ganhando novos significados ao lon- 
go do tempo. Sua aplicação difundiu-se tão amplamente, e sua utilização atingiu áreas tão diversas, que esses diferentes significados, hoje, pouco têm a ver uns com os outros.

Fora do campo da lógica, a idéia de identidade foi inicialmente aplicada a algo descrevendo o indivíduo ou a ele limitado. Hoje, porém, muitos campos do conhecimento, fora dos limites das ciências sociais ${ }^{7}$ e dentro deles - como é o caso atualmente da análise organizacional - têm aplicado o conceito de identidade a outros objetos, como grupos sociais ou religiosos, nações, a espécie humana etc.

A noção psicanalítica de identidade individual foi provavelmente a primeira e mais influente utilização do conceito nas ciências sociais. Freud, por exemplo, usou a expressão "identidade interior" para descrever a razão pela qual tinha sido tão atraído ao Judaísmo: " $O$ que me ligou ao Judaísmo não foi (e me envergonho de admitir) nem fé nem orgulho patriota,

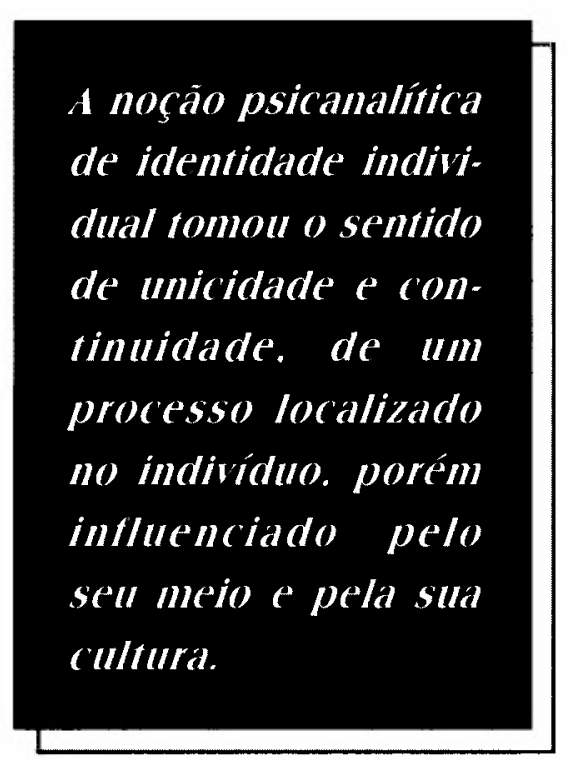

uma vez que eu sempre fui um incrédulo $e$ fui criado sem qualquer pensamento religioso (...). Porém, muitas outras coisas permaneceram para fazer a atração ao Judaísmo $e$ aos judeus irresistível - muitas forças emocionais obscuras, que eram mais poderosas quanto menos podiam ser expressas em palavras, assim como uma clara consciência de identidade interior, a segura privacidade de uma construção mental comum" .8

Embora se afirme que essa foi a única ocasião em que Freud usou o termo identidade de forma mais do que casual, ${ }^{9}$ a idéia de tais "obscuras forças emocionais" e sua relação com o ego tornaram-se pontos de partida para diversos usos futuros do termo.

Uma vez que a relação entre os conceitos de identidade, self e ego parece ser muito importante para utilizações posteriores da idéia de identidade, é útil clarificar seus significados.

Os termos “identidade" e self serão usados aqui com significados similares (exceção feita ao conceito jungiano de self). Entretanto, devemos deixar claro que essas duas idéias não são iguais. O termo "identidade" deriva dos vocábulos latinos idem e identitas (ambos significando "o mesmo") e do vocábulo entitas (entidade). Ao combinar essas raízes, identidade poderia então significar "a mesma entidade". Alguns autores já sugeriram que a palavra pode também estar associada a outro vocábulo latino, identidem, significando "repetidamente", "uma e outra vez". ${ }^{10}$ Já a origem da palavra self não é tão clara. Presume-se, com frequiência, que ela é uma composição ligada ao prenômio indo-europeu se-, significando "o eu de cada um"."1 A partir desses significados, parece ser possível sugerir que, enquanto identidade refere-se a um conteúdo ou propriedade, o self pode representar a entidade que a incorpora. Por sua vez, ego, o vocábulo latino equivalente a "eu", é definido como a concepção que a pessoa faz de si mesma. Na psicanálise, ego é algo que se mantém razoavelmente estável durante a vida e relaciona-se a uma série de funções da personalidade que permitem ao indivíduo lidar com a realidade. ${ }^{12}$ Freud usou o conceito de ego como um dos agentes em seu modelo da psique, para referir-se a um meio consciente e racional que seria, por um lado, forçado pelos impulsos do id e, por outro, reprimido pelo controle do superego.$^{13}$ Depois de Freud, o conceito de ego foi expandido, dentro e além dos limites da psicanálise. ${ }^{14}$

A noção psicanalítica resultante do conceito de identidade foi amplamente popularizada por teóricos do campo hoje conhecido como Psicologia Diferencial, em particular por Erik Erikson. Psicanalista influente, Erikson introduziu e divulgou, nas ciências comportamentais, o termo "crise de identidade". Trabalhando em uma clínica de reabilitação, durante a Segunda Guerra Mundial, Erikson e seus colegas observaram o seguinte: " $A$ maioria de nossos pacientes (...) tinha perdido, pelas exigências da guerra, um sentido de
7. Ver CARR, B. Metaphysics: An introduction. Basingstoke: Macmillan, 1987.

8. Ver FREUD, $S$. Address to the Society of B'nai B'rith. The Standard Edition of the Complete Psychological Works of Sigmund Freud v.20, p. 273-274. London: Hogarth Press, 195 [1926]

9. Ver ERIKSON, E. Identity, youth and crisis. New York: W W. Norton \& Company, 1968, $p$. 21.

10. Ver ONIONS, C. T. (Ed.) The Oxford Dictionary of English Etymology. Oxford: Oxford University Press, 1979.

11. Ver BARNHART, R. K. (Ed.) The Barnhart Dictionary of Etymology. New York: H. W. Wilson Company, 1988.

12. Ver LAPSLEY, D. K. \& POWER, F. C. (Eds.) Self, Ego, and ldentity: Integrative approaches. New York: Springer-Verlag, 1988.

13. Ver FREUD, S. The Ego and the Id. The Major Works of Sigmund Freud. Chicago: William Benton/Britannica Inc., 1952 [1923]

14. Exemplos dessa expansăo incluem FREUD, A. The Ego and the Mechanisms of Defense. New York: International Universities Press. 1967; ERIKSON, E. Op. cit:; ERICKSON, E. Iden tity and the life cycle. New York: W.W. Norton\&Company, 1980. 
15. Ver ERIKSON, E. Op. cit., 1968, p. 17-19

16. Ver WOOLEY, T. Self identity: the neglected resource. Paper apresentado na XIII SCOS. Standing Conference on Organizational Symbolism. Turku, Finlândia, 1995 e DIAMOND M. A. The Unconscious Life of Organizations Interpreting organizatlonal identity. Westport: Quorum Books, 1993

17. ERIKSON, E. Op. cit., 1980, p. 109.

16. WOOLEY, T. Op. cit unicidade pessoal e de continuidade histórica. Eles tinham sido privados daquele controle central sobre si mesmos pelo qual, no esquema psicanalítico, somente o 'agente interior' do ego poderia ser responsabilizado. Portanto, nós falavamos da perda da 'identidade do ego'."15

Assim, a noção psicanalítica de identidade individual tomou o sentido de unicidade $e$ continuidade, de um processo localizado no indivíduo, porém influenciado pelo seu meio e pela sua cultura. ${ }^{16}$ Colocado de outra maneira, o conceito mais popularizado de identidade define-a como uma classificação do self que expressa o indivíduo como reconhecidamente diferente dos demais, e como similar a membros da mesma classe. ${ }^{17}$

Parece ter sido esse o sentido de identidade individual que foi mais utilizado para explicar processos e elucidar características de outros objetos, em outros campos das ciências humanas além da Psicologia. Dessa forma, tornouse usual aplicar o termo identidade a entidades plurais, como grupos étnicos, organizações, nações etc.

Entretanto, cada campo científico, escola de pensamento e perspectiva intelectual tem-se apropriado da idéia de identidade à sua maneira, procurando definir o conceito segundo seus próprios objetivos e interesses. ${ }^{18}$

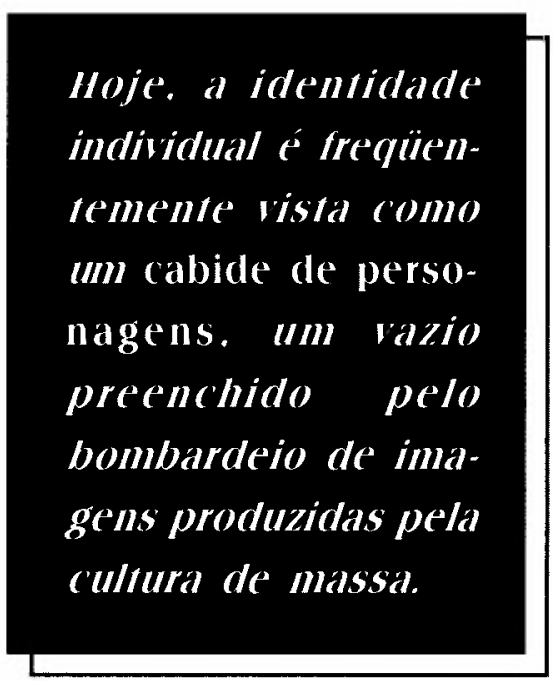
Cada uma dessas trilhas de análise gerou seus próprios seguidores que, por sua vez, criaram - inclusive na análise das organizações definições de identidade relativamente distintas entre si. A seguir, procuraremos tornar o conceito de identidade mais tratável, buscando viabilizar seu uso na análise das organizações.

fornecer um instrumento didático para mapear as principais correntes existentes no estudo da identidade.

\section{As duas dimensões básicas}

A primeira dimensão - dimensão do objeto focal - distingue as perspectivas existentes sobre identidade através da diferenciação do objeto sobre o qual o conceito é utilizado. Além do objeto indivíduo (o foco mais popular), outras entidades também podem "possuir identidade". Essa primeira dimensão diferencia as abordagens que enfocam identidade do indivíduo, do grupo, da organização, da humanidade etc. É conveniente notar que os objetos focais assinalados nessa dimensão - imaginada como continuum não são completamente separados, havendo entre eles interações e interpenetrações.

A segunda dimensão - dimensão da observação - distingue conceitos de identidade formulados a partir de diferentes pontos de observação. Qualquer que seja o objeto analisado, sua identidade pode ser observada interna ou externamente. Tomemos como exemplo a identidade individual: em um extremo, poderíamos referir-nos a esse objeto focal a partir de algo que apontaria para aquilo que, na pessoa, define a linha divisoria entre o eu e o não-eu: seu self. No outro extremo estaria a noção que os outros têm do que a pessoa é: sua imagem. Entre esses dois extremos estaria a expressão exterior da pessoa: seu comportamento e a percepção da pessoa sobre si mesma: sua autopercepção.

\section{Um primeiro quadro conceitual}

A combinação dessas duas dimensões re-

\section{DESENVOLVIMENTO DE UM PRIMEIRO QUADRO CONCEITUAL}

Nesta seção, propomos um quadro conceitual desenvolvido a partir da sobreposição de duas dimensões: a dimensão do objeto focal e a dimensão da observação. O objetivo é sulta em um plano - ou quadro conceitual onde é possível localizar as diversas abordagens do conceito de identidade. Neste trabalho, o quadro conceitual (ver figura 1) resultou da combinação de quatro objetos focais indivíduo, grupo, organização e humanidade - e quatro níveis de observação - identida- 
de interior (ou self), comportamento, autopercepção e imagem.

As células resultantes dessa sobreposição distinguem, por exemplo, a perspectiva que enfoca identidade como a imagem que uma pessoa tem no seu grupo (individuo como objeto focal, identidade observada externamente), daquela que entende como identidade o autoconceito organizacional (organização como objeto focal, identidade observada por autopercepção).

É conveniente lembrar que o estudo da identidade é dinâmico e complexo e que o quadro conceitual apresentado é um recurso didático com certas limitações. Sua apresentação, por exemplo, pode transmitir a idéia de espaços - ou células - bem delimitados, com fronteiras claras e conteúdo teórico sólido. Entretanto, muitos deles interpenetram-se, enquanto outros espaços estão praticamente inexplorados. Além disso, à medida que o conceito de identidade foi-se expandindo de um campo científico a outro, a diversidade teórica aumentou, originando ora transposições diretas de conceitos, ora conceitos distintos.

No decorrer do texto, percorreremos o quadro conceitual abordando: (1) a evolução histó- rica do estudo do conceito de identidade; (2) as interligações entre quadrantes; e (3) as áreas - e células - ainda não preenchidas por teoria.

\section{Evolução do estudo da identidade}

Para melhor analisar como evoluiu ao longo do tempo o estudo da identidade, agrupamos - áreas hachuradas ou "nebulosas" as células mais povoadas, reunindo contribuições que, apesar de distintas, contêm muitos pontos em comum. É conveniente lembrar que a formação desses agrupamentos também foi fruto dos objetivos deste estudo, havendo, portanto, certo grau de arbitrariedade. Dessa forma, o espectro resultante é necessariamente incompleto, mas didaticamente poderoso, à medida que indica as grandes tendências que existem em torno da idéia de identidade, bem como as ligações conceituais entre essas tendências. Ao percorrer esse caminho evolutivo, o quadro parece ganhar mais dinamismo e coerência.

O primeiro agrupamento (ver área (1) na figura 1) reúne os estudos pioneiros de identidade individual de Erikson - já mencionados com sua origem psicanalítica e, em especial,

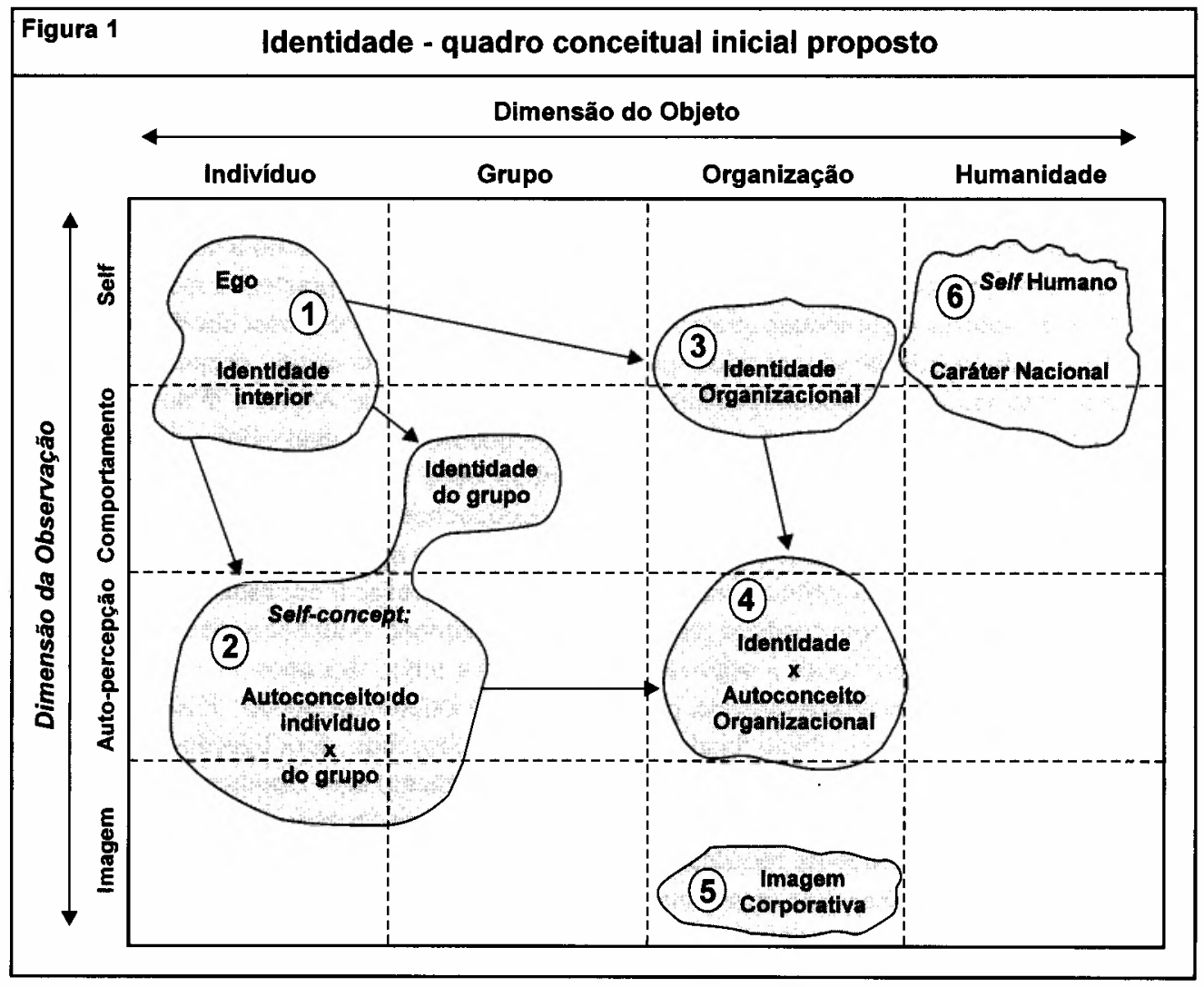

$R A E \cdot$ v. $37 \cdot$ n. $1 \cdot$ Jan./Mar. 1997 
19. Apesar de o trabalho de Erikson não ter de fato originado todas as perspectivas sobre identidade individual que há em outros campos do conhecimento (muitas outras abordagens existiam antes da sua, por exemplo, em Psicologia Social), a popularização da sua pesquisa definitivamente popularização da sua pesquisa definitivamente deu novo fólego e chamou a atenção para o conceito de identidade em diversas disciplinas dentro das ciências sociais.

20. Ver STRAUSS, A. Mirrors and Masks: The search for identity. Glencoe, IL: The Free Press, 1959 e GOFFMAN, E Asylums: Essays on the social situation of mental patients and other social situation of mental patients and other inmates. New York: Anchor Books, 1961. GOFFMAN, E. Stigma: Notes on the management of the spoiled identity. Hardmon: Pen-
guin, 1968 .

21. Ver GECAS, V. The self-concept. Annual Review of Sociology, v. 8, p. 1-33, 1982; TAJFEL, $H$. Social psychology of intergroup relations. In: ROSENWEIG, M. R. \& PORTER, L. W (Eds.), Annual Review of Psychology, v. 33, p. 1-39. Palo Alto: Annual Reviews, 1982; TAJFEL, H. \& TURNER, J.C. The social identity theory of intergroup behavior. In: WORCHEL O AUSTIM W G (EDs). PsyWORCHEL, S. Q AUSTIN, W. G. (Eds.), Psy24. Chicago: Nelson-Hall Publishers, 1985.

22. Ver BROWN, R. \& WILLIAMS, J. Group identification: the same thing to all people? Human Relations, v. 37, p. 547-564, 1984; O'REILLY, C. \& CHATMAN, J. Organizational commitment and psychological attachment. Joumal of Applied Psychology, v. 71, p. 4965, 1986; CHATMAN, J. Matching people and organizations. Administrative Science Quarterly, v. 36, p. 455-484, 1991

23. Ver KRAMER, R. M. \& BREWER, M. B. EFfects of group identity on resource use in a
simulated common dilemma. Journal of $P e r-$ sonality and Social Psychology, v. 46, p. 1044-1057, 1984

24. Ver ALBERT, S \& WHETTEN, D. A. Organizational identity. In: CUMMINGS, L. L. \& STAW B. M. (Eds.), Research in Organizational Behavior, v. 7. p. 263-295. Greenwich, CT: JAI Press, 1985.

25. Ver ALBERT, S. \& WHETTEN, D. A. Op. cit., p. 265.

26. Ver WHETTEN, D. If it isn't 'core, enduring, and distinctive' it isn't identity. Paper apresentado em simpósio sobre identidade organizacional (Beyond 'central, enduring, and distinctive': Reconceptualizing organizational identity, MOC/OB Divisions), durante as reuniōes anuais da Academy of Management. Vancouver, B.C. (Canadá), 1995

27. Ver HATCH, M. J. \& SCHULTZ, M. From tribes to texts: Cultural impressions of image and identity. Paper apresentado na XIII SCOS - Standing Conference on Organizational Symbolism. Turku, Finlândia, 1995; WHETTEN, Symbolism. Turku, Finlândia, 1995; WHETTEN,
D. \& GREGERSEN, H. Social identity and charD. \& GREGERSEN, H. Social identity and char-
acteristics of group categories and boundaries. acteristics of group categories and boundaries. Conference on Organizational Symbolism. Turku, Finlândia, 1995; ALBERT, S. \& WHETTEN, D. A. Managing organizations with a dual or hybrid identity. Paper apresentado em simpósio sobre identidade organizacional (Managing Organizational Identities and Image, MOC/OMT Divisions), durante as reuniōes anuais da Academy of Management. Vancouver, B.C. (Canadá), 1995.

28. Ver HATCH, M. J. The dynamics of organizational culture. Academy of Management Review, v. 18, p. 657-693, 1993

29. Ver DUTTON, J. E. \& DUKERICH, J. M. Keeping an eye on the mirror: the role of image and identity in organizational adaptation. Academy of Management Journal, v. 34, 366-375, 1991; DUTTON, J. E., DUKERICH, J. M., HAROUAIL, C. V. Organizational images and member identification. Administrative Science Quarterly, v. 39, p. 239-263, 1994. com a influência do conceito Freudiano de $e g o$. A partir desse primeiro agrupamento, cuja "nebulosa" abrange espaços dos quadrantes de identidade individual - self - e de identidade individual expressa - comportamento - o conceito de identidade expandiu-se para diversos campos do conhecimento, especialmente pela popularização do trabalho de Erikson durante os anos $70 .^{19}$

O segundo agrupamento (ver área (2), na figura 1) abrange diversos estudos clássicos e contemporâneos em Psicologia Social que (a) referem-se à identidade como autoconceito (ou self-concept, isto é, o conceito que uma entidade - um grupo, por exemplo - ou que uma pessoa faz de si mesma); ou (b) relacionamidentidade individual e identidade grupal, em especial através do conceito de "identificação". Neste segundo agrupamento, como um todo, tanto os estudos clássicos, ${ }^{20}$ quanto os contemporâneos ${ }^{21}$ tendem a entender identidade como um fenômeno social, que deriva dos significados que indivíduos atribuem à sua interação com múltiplos grupos sociais durante as suas vidas.

$\mathrm{Na}$ linha de pesquisa que lida com identificação, diversos estudos sustentam que uma parte significativa da identidade do indivíduo é definida pelo grupo ao qual ele pertence; ou seja, a forma e o nível do sentido de pertencimento do indivíduo a um grupo social molda seu autoconceito. Seguindo esse tipo de raciocínio, mas em uma outra vertente derivada da raiz da Psicologia Social, alguns autores argumentam que a identidade do indivíduo também está relacionada ao grau de identificação que os membros têm com a organização. ${ }^{22}$ Por fim, outros estudiosos argumentam que a identidade é um atributo que não só indivíduos possuem, mas também grupos podem possuir. ${ }^{23}$

O que todos esses autores e perspectivas têm em comum é o fato de entenderem identidade como um atributo sócio-cognitivo. Isso significa ao menos duas coisas: primeiro, que para eles, identidade não é apenas um atributo exclusivo e inato ao indivíduo: o grupo também passa a ser percebido como objeto focal; e segundo, a identidade passa a ser vista reflexivamente: pode referir-se também à forma do objeto focal ver a si mesmo. Como um todo, as diversas ramificações desse agrupamento formam um dos campos mais influentes no estudo de identidade.

O terceiro agrupamento (ver área (3) na figura 1) deriva em grande parte do primeiro ou seja, do conceito de identidade individual - mas não ficou indiferente às conotações de identidade usadas na Psicologia Social em anos recentes. Esse grupo reúne as concepções mais clássicas de identidade que surgiram no âmbito organizacional, a partir do trabalho pioneiro de Albert \& Whetten. ${ }^{24}$ A partir das noções de Erikson sobre identidade individual e dos primeiros trabalhos sobre cultura e simbolismo organizacional, esses autores sugeriram que organizações também possuem uma identidade, ou um "caráter central". Albert e Whetten propõem três critérios - hoje muito populares na literatura - para definir a identidade de uma organizacão. Assim, a identidade organizacional compreenderia as crenças partilhadas pelos membros sobre o que é (a) central, (b) distintivo, e (c) duradouro na organização. O critério de centralidade aponta as características vistas como a essência da organização. $O$ critério de distintividade aponta os elementos que distinguiriam uma organização das outras com as quais poderia ser comparada. Por fim, o critério de continuidade temporal ressalta as características estáveis no tempo. Esses três critérios seriam, segundo os autores, "cada um necessário e, como um conjunto, suficientes" para definir "identidade organizacional" como conceito científico. ${ }^{25}$

Ainda nesse terceiro agrupamento, podemse encontrar trabalhos mais recentes que procuram rever o conceito tradicional de identidade organizacional, ${ }^{26}$ apresentar modelos alternativos ou complementares, ${ }^{27}$ ou ainda diferenciar a idéia de identidade organizacional de outros conceitos, como o de cultura organizacional. ${ }^{28} \mathrm{O}$ que caracteriza mais claramente esse agrupamento é o vínculo do conceito de identidade com o campo organizacional que, pela influência de Albert e Whetten, ocorreu principalmente nas duas células do quadro conceitual mais ligadas a self e comportamento (organizacionais).

O quarto agrupamento (ver área (4) na figura 1) tem origem em estudos de identidade organizacional, realizados desde o final dos anos 80 e início dos anos 90, a partir de conceitos de Psicologia Social. Esse quarto agrupamento tem uma clara ligação com o segundo agrupamento aqui mencionado. As correntes nessa nova "nebulosa" têm em comum a idéia de identidade organizacional como uma função da forma pela qual a organização percebe a si mesma. Autores nessa linha de pesquisa têm tido grande influência no campo. ${ }^{29}$ 
Esse quarto agrupamento tem-se tornado cada vez mais relevante também por ter estabelecido uma ligação entre os estudos de identidade no terreno organizacional e no campo da Psicologia Social. Como fruto dessa ligação, surgiram pesquisas que abrangem conceitos de identidade individual, grupal e organizacional. Essas pesquisas incluem: (1) a discussão da relacão entre identificação organizacional e autoconceito individual $;^{30} \mathrm{e}$ (2) a análise da relação entre imagem do grupo ou da organização com a identificação do individuo no grupo. ${ }^{31}$

Recentemente, dois outros campos de pesquisa têm sido desenvolvidos paralelamente a esse quarto agrupamento. Eles são importantes para sinalizar futuras direções de pesquisa do conceito de identidade no campo organizacional e estão aqui identificados como o quinto e o sexto agrupamentos.

$O$ quinto agrupamento (ver área (5) na figura 1) discute o conceito de identidade organizacional de forma muito mais instrumental, dentro da esfera da chamada "imagem corporativa". Nesse grupo, o foco de pesquisa é a compreensão de como as organizações administram a sua imagem externa e como tal imagem afeta-as. ${ }^{32}$

Ainda nesse agrupa-

mento, alguns outros autores analisam de modo crítico a manipulação da imagem e da identidade corporativa pelas empresas. ${ }^{33}$ Como veremos na próxima seção, diversos deles realizam essa crítica a partir de idéias dos teóricos pós-modernistas.

O sexto agrupamento (ver área (6) na figura 1) compreende as pesquisas que discutem, dentro e fora da área organizacional, conceitos de identidade em nível macro, seja de nações ${ }^{34}$ - como objeto focal - ou da humanidade. ${ }^{35}$ Neste grupo, uma tendência de pesquisa, também influenciada por idéias pós-modernas, parece levar a questionamentos sobre a efetiva existência e sobre a natureza de um self humano.
A análise da evolução dessas diversas correntes pode demonstrar a utilidade didática do quadro conceitual inicial. Este permite também distinguir perspectivas de identidade que já existem em campos distintos e que poderiam ser confundidas em uma análise mais superficial. Enfim, permite identificar os padrões evolutivos do estudo acerca de identidade e os campos de pesquisa a serem explorados. Através do quadro pode-se observar, por exemplo, como a pesquisa sobre identidade tem caminhado no sentido do individual para o coletivo, e do ponto de observação interno para o externo. Nesse movimento, muitas células permanecem inexploradas, como a discussão de uma identidade interior no âmbito do grupo e da organização, ou a questão da imagem do grupo e do indivíduo (apesar da existência do trabalho clássico de Goffman ${ }^{36}$ neste sentido).

Neste ponto do trabalho, consideramos oportuno retornar à questão das limitações do quadro conceitual. A primeira dessas limitações é que algumas perspectivas inteiras não podem ser bem representadas no modelo. A noção Jungiana de self, por exemplo, permeia diversas células no nível da identidade $o b$ servada internamente, alternando-se ora ligada ao objeto indivíduo (aproximando-se aí dos conceitos de self e ego Freudiano), ora relacionada ao objeto humanidade (aproximando-se do self humano ${ }^{37}$ ).

A segunda limitação está na própria natureza dos agrupamentos. Embora, como assinalamos anteriormente, esses agrupamentos reúnam abordagens e vertentes afins, não podemos deixar de notar os antagonismos intra-agrupamentos, próprios de um campo diverso e fragmentado como é este da identidade. A terceira e mais importante limitação, relacionada ao pressuposto básico do quadro - ou seja, à própria definição de identidade - será tratada na seção seguinte.
30. Ver KRAMER, R. M. Organizational identification and cooperation. In: MURNIGHAN, $K$ Ed) Social psychology in organizations: AdEd.) Social psychology in organizations. Advances in theory and practice. Englewood valits, in theory and practico, Engelly \& CHATMAN, Op. cit., ASHFORTH, B. E. \& MAEL, F. Social identity theory and the organization. Academy of Management Review, v.14, D. 2039,1989

31. Ver DUTTON, J. E., DUKERICH, J. M., HARQUAIL, C. V. Op. cit.; ELSBACH, K. D. \& KRAMER, R. M. Cognitive strategies for managing organizational identity: a study of busiand ness school responses to the Business Week rankins. Paper apresentado durante as D Dallas, TX, 1994; KRAMER, R.M. Op. cit.

32. Ver BLASICK, J. The communication of organizational identity: a source of competitive advantage. Paper apresentado durante as reuniöes anuais da Academy of Management. Cincinnati, OH, 1996; FOMBRUN, C. \& SHANLEY, $M$. What's in a name ? Reputation bullding and corporate strategy $A c a d e m y$ of $M a n a g e m e n t$ corporate strategy. Academy of Management Joumal, v. 33, p. 233-258, 1990; GARBET, T. How to build a corporation's identity and project its image. Lexington, MA: D.C. Health
and Company, 1988 .

33. Ver ALVESSON, $M$ Organization: From substance to image? Organization Studies, v. 11, n. 3, p. 373-394, 1990; GIOIA, D. \& SCHULTZ, M. Adaptive instability: the interrelationship between identity and image in organizations. Paper apresentado em simpósio sobre identidade organizacional Managing Organizational Identities and Image, MOC/OMT Divisions), durante as reuniōes anuais da Academy of Management Acande) of Mancouver, B.C. Op. cit., 1995 , HATC,M. J. \& SCHULTZ, M.

34. Ver HOFSTEDE, G. Culture's Consequences: International differences in workrelated values. Beverly Hills, CA: Sage, 1980; HOFSTEDE, G. Measuring organizational cultures: A qualitative and quantitative study across twenty cases. Administrative Science Cuarterly, v. 35, n. 2, p. 286, 1990

35. Ver BRONOWSKI, J. The identity of man. Garden City, NY: Doubleday, 1972 Business Week. The virtual corporation. February 8, p. 36-41, 1993.

36. GOFFMAN, E. Op. cit., 1968.

37. Ver JUNG, C. G. Aion: researches into the phenomenology of the Self. In: Collected Works, v. 9, Part II, p. 1-42. Princeton, NJ: Princeton University Press, 1959; JUNG, C. G. The structure and dynamics of the psyche. In: Collected Works, v. 8. p. 283-342. Princeton, NJ: Princeton University Press, 1960. 


\section{AMPLIANDO O QUADRO CONCEITUAL}

Na seção anterior desenvolvemos um quadro conceitual a partir de duas dimensões: a dimensão do objeto e a dimensão do observador. Nele localizamos diversas contribuições teóricas. Pudemos, assim, analisar diferentes vertentes de estudo dentro do campo e explorar algumas ligações entre elas. Pretendemos agora ampliar e aprofundar a discussão sobre o conceito de identidade a partir do questionamento do pressuposto básico que norteou a construção do quadro conceitual inicial. Esse pressuposto é essencial porque fundamenta ou norteia todas as outras definições constantes do quadro, e diz respeito à própria noção de identidade como algo central, distintivo e duradouro.

Parece-nos natural, e até obrigatório, incluir aqui esse questionamento, em função de sua presença explícita ou implícita em muitos debates contemporâneos. A existência de uma identidade individual, por exemplo, é objeto constante de análise em Psicologia Social e em Estudos Culturais, especialmente entre pensadores pós-modernistas. Do lado organizacional, é a tendência de quebra de fronteiras, ou virtualização, que impõe o debate. A questão que se coloca aqui é a seguinte: será a noção de identidade como algo central, distintivo e duradouro compatível com as novas configurações organizacionais?

Para discutir estas questões, introduziremos um terceiro eixo no quadro conceitual: o eixo da definição de identidade. Em segui$\mathrm{da}$, tomaremos dois epi-quadrantes do quadro anterior: o da identidade individual e o da identidade organizacional, e realizaremos uma análise das implicações dessa nova perspectiva sobre os conceitos.

\section{A dimensão da definição de identidade}

O objetivo da inclusão desse terceiro eixo é dotar nosso quadro conceitual de uma dimensão epistemológica e ontológica adicional. Esse terceiro eixo é um eixo triplo, pois parte dos três parâmetros de definição de identidade - o ser central, distintivo e duradouro - e dos seus opostos - o poder ser fragmentado, não distintivo e efêmero (ver figura 2).

A figura 2 representa o quadro conceitual desenvolvido nesta parte do trabalho como um plano que se desloca ao longo do terceiro eixo. A posição relativa do quadro ou de cada célula no eixo é o objeto da discussão que realizaremos a seguir.

É importante observar que, embora na discussão que se segue apliquemo-lo a apenas dois objetos focais - o da identidade individual e o da identidade organizacional - esse terceiro eixo é válido para qualquer um dos demais quadrantes. Assim, se tomarmos, por

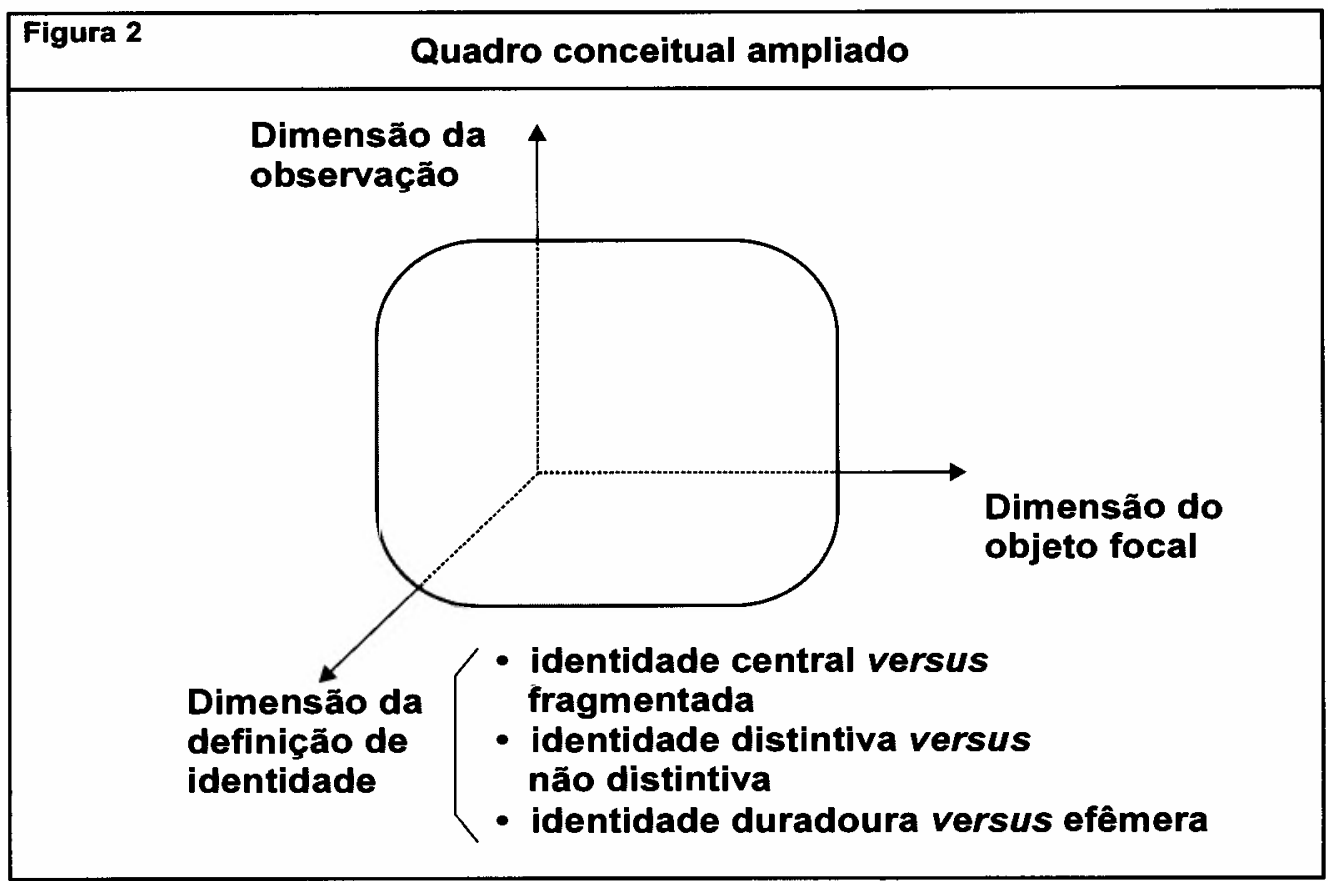


exemplo, a célula da imagem individual, poderíamos discutir sobre sua centralidade ou fragmentação, sua distintividade ou indistintividade e sobre sua durabilidade ou volatilidade. $\mathrm{O}$ mesmo vale para a autopercepção individual, o comportamento organizacional, a imagem do grupo etc.

\section{A identidade individual $e$ espirito da época}

A trajetória humana é um contínuo repensar-se. O ser humano já foi definido como uma sombra, um sopro divino ou uma alma habitando um corpo. Na Idade Média, o conceito de identidade individual não existia e a ordem do universo era uma dádiva de Deus. ${ }^{38}$ Com o Cartesianismo e o

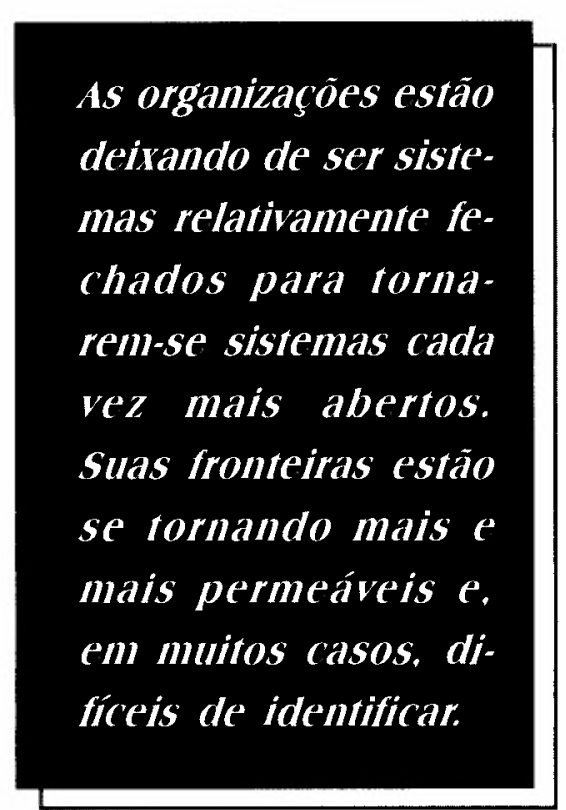

formação. A conclusão é que a identidade moderna é múltipla: um self que corresponde a um contexto social-plural. Na sua obra, Musil declara que cada indivíduo contém, no mínimo, nove personagens ligados a: vocação, nação, estado, classe social, contexto geográfico, sexualidade, consciente, inconsciente e vida privada. Musil também afirma que pode haver um décimo personagem, ligado à capacidade humana para gerar utopias e atingir um sonho de um selfrestaurado à unidade.

$A$ análise de Berger mostra que a identidade autônoma, se alguma vez chegou a existir, foi apenas como possibilidade teórica. Na realidade, a emergência do conceito de identidade Iluminismo, essas formulações cederam lugar à visão do homem como ser racional. ${ }^{39}$ Essa nova era trouxe também, implicitamente, a crença na possibilidade de construção de uma identidade autônoma.

Hoje, contudo, essa crença está abalada e a identidade individual é freqüentemente vista como um cabide de personagens, um vazio preenchido pelo bombardeio de imagens produzidas pela cultura de massa. A identidade não é mais vista exclusivamente como uma entidade autônoma, estática e duradoura, mas como um processo de construção, uma atividade humana, mediada pelo uso da linguagem e ligada à socialização do indivíduo por meio da interação simbólica com seu meio. Nessa perspectiva, a origem da identidade individual está na comunidade, nos artefatos culturais e instituições que a comunidade propicia. ${ }^{40}$ Dessa forma, durante a existência de um indivíduo, a identidade pode ser adquirida e perdida, passando por períodos de autenticidade e falsidade.

Berger $^{41}$ realiza uma interessante análise da obra de Robert Musil Der Mann ohne Eingenshaften (O Homem sem Qualidades), acompanhando a trajetória dos personagens principais sob o prisma da tentativa de construção da identidade em um mundo em trans- está associada não só ao desaparecimento da sociedade feudal, mas também à comoditização dos sentimentos e à utilitarização do ser humano, trazidos pelo capitalismo. Assim, se um self verdadeiro for realmente possível, somente poderemos atingi-lo através de um enorme esforço de autodesenvolvimento.

\section{Fronteiras, virtualidade e identidade organizacional}

A maioria das teorias em Estudos Organizacionais pressupõem organizações como entidades distintas, com ativos mensuráveis, prédios, estruturas definidas, mão-de-obra fixa e fronteiras claras. ${ }^{42}$ Enquanto isso, no mundo real, multiplicam-se terceirizações, contratações de mão-de-obra temporária, parcerias com fornecedores e clientes, assim como alianças com concorrentes. As organizações estão deixando de ser sistemas relativamente fechados para tornarem-se sistemas cada vez mais abertos. Suas fronteiras estão se tornando mais e mais permeáveis e, em muitos casos, difíceis de identificar. ${ }^{43}$

Ashkenas, Ulrich, Jick \& Kerr ${ }^{44}$ argumentam que as fronteiras organizacionais estão sendo quebradas em quatro níveis: (1) vertical - com o enfraquecimento das hierarquias;
38. Ver DOWD, J. J. Social Psychology in a postmodern age: a discipline without a sub. ject. Department of Sociology, University of Georgia, Athens, GA, 1992.

39. Ver WEIGERT, A. J., TEIGTE, J. S. \& TEIGTE, D. W. Society and identity: toward a sociological psychology. Cambridge, England: Cambridge University Press, 1986

40. Ver FRANKS, D. The self in evolutionary perspective. Studies in Symbolic Interaction, Supplement 1, p. 29-61, 1985; WEIGERT, A.J. et al., op. cit.

41. Ver BERGER, P. Robert Musil and the salvage of the self. Partisan Review, v. LI, p. 638vage of the
$650,1984$.

42. Ver THORNTON, P. H. \& TUMA, N. B. The problem of boundaries in contemporary research on organizations. Academy of Manage ment Proceedings. Vancouver, B. C. (Canada), 1995

43. Ver STRATI, A. Aesthetics and organizations without walls. Studies in Culture, Organizations and Socleties, v.1, n. 1, p. 83-105, 1995.

44. Ver ASHKENAS, R., ULRICH, D., JICK, T. \& KERR, S. The boundaryless organization: Breaking the chalns of organizational struc. Breaking the chalns of organizational st
ture. San Francisco: Jossey-Bass, 1995. 
45. Ver BUSINESS WEEK The virtual corporation. February 8, p. 36-41, 1993; GRENIER R. \& METES, G. Going virtual: moving your organization into the 21st. century. Upper Saddle River, New Jersey: Prentice-Hall, 1995 ASHKENAS, R., ULRICH, D., JICK, T. \& KERR S. Op. cit.

46. Ver HATCH, M. J. \& SCHULTZ, M. An interdisciplinary framework for studying organizational culture, identity and image in postindustrial organizations. Paper apresentado durante as reuniöes anuais da Academy of Management Cincinnati, OH, 1996: STRATI A OD cit: CLANCY T T. OH.

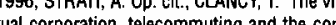
cot of ecutive, $\times$, 8.2 , 0. 7-10, 1994

47. Ver HATCH, M. J. \& SCHJLTZ, M. Op. cit. 1995.

48. Ver MANTOVANI, G. Virtual reality as a communication environment: consensual hatlucination, fiction, and possible selves. Human Relations, v. 48, n. 6, p. 669-683, 1995.

49. Ver HEDBERG, B. Imaginary organizations. Stockholm: Stockholm University (Internal Document), 1994.
(2) horizontal - com o foco nos processos;

(3) externo - com a aproximação com fornecedores e clientes; e (4) geográfico - com a expansão mundial dos negócios.

As implicações desse quadro de transformações têm sido objeto de reflexão apenas recentemente, tanto para executivos e gerentes, ${ }^{45}$ quanto para teóricos organizacionais. ${ }^{46}$ Algumas vertentes do campo da Cultura Organizacional, por exemplo, costumam tratar o objeto de estudo organização como a Antropologia do século passado tratava as tribos primitivas, ou seja, como um sistema fechado, com identidade e fronteiras definidas. Nossa realidade, ao contrário, mostra o fim das fronteiras entre relações internas e externas. As tribos organizacionais tornaram-se entidades fragmentadas; sua coerência foi despedaçada e substituída por uma multiplicidade de significados e interpretações. ${ }^{47}$

Enfim, continua fazendo sentido falar em identidade organizacional nesse contexto? Visando responder essa questão, gostaríamos de

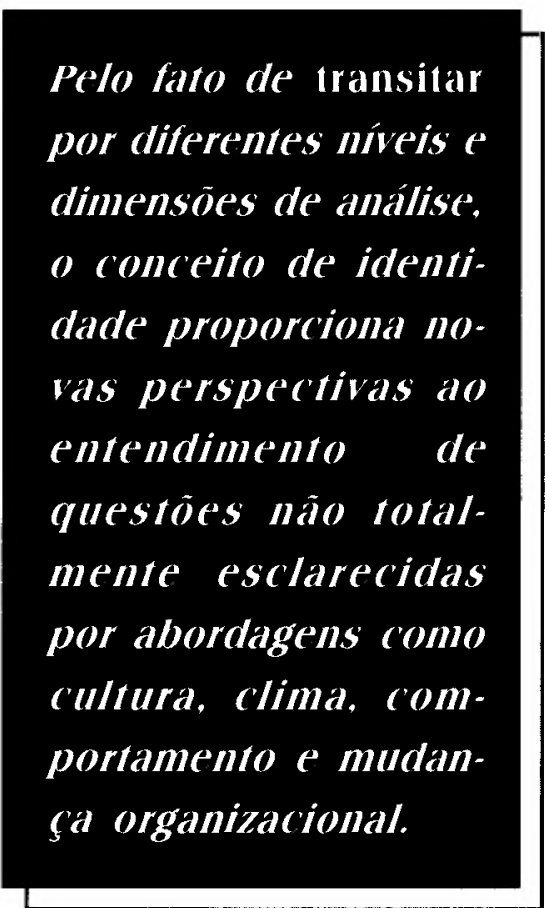

eletrônica. No ciberespaço, uma pessoa pode construir novos papéis, identidades ficcionais. Assim como a realidade virtual é um palco para a atuação dos atores individuais, a organização virtual é um palco para a interação dos atores organizacionais.

Segundo Hedberg, ${ }^{49}$ uma organização virtual - ou imagética - ocorre onde importantes valores, atores e processos existem e funcionam fora da terra firme da jurisdição da empresa. Eles vivem uma meia-vida, uma quase miragem entre o real e o não real. Por isso o termo organização imagética. Essas organizações identificam oportunidades de mercado e mobilizam rapidamente recursos, utilizando alianças temporárias, combinando competências independentemente de fronteiras e empregando maciçamente a tecnologia de informações.

Então, que conclusões poderíamos tirar de todas essas tendências? Talvez seja mais próprio repensar as questões, pois as categorias nelas mencionadas fofocalizar mais detalhadamente o conceito de organização virtual, um tipo de organização sem fronteiras.

Para entender o fenômeno emergente das organizações virtuais, é necessário desenvolver um novo vocabulário e um novo corpo conceitual. Então, começaremos por clarificar o termo realidade virtual, uma idéia que surgiu e foi popularizada na década de oitenta. A realidade virtual relaciona-se à possibilidade de o computador criar imagens animadas em tempo real. Segundo Mantovani, ${ }^{48}$ a simples existência do termo obriga-nos a rever o que consideramos como realidade. A realidade virtual é um paradoxo em termos de experiência sensorial. Ela cria um espaço de interação onde a presença física é evidente mas as identidades são evasivas e efêmeras. Essa interação é realizada por projeções, simulacros criados e condicionados por mídia ram criadas para um modelo de organização e uma forma de análise organizacional em extinção. Especificamente quanto ao conceito de identidade organizacional, se essa tendência - que envolve tanto alianças entre corporações como tendências virtualizantes intracorporações e entre pequenas empresas e trabalhadores individuais (teletrabalho) - é realmente importante, há necessidade de repensar-se completamente o conceito.

Em organizações virtuais, a identidade só pode ser vista como algo fragmentado, não distintivo e efêmero. Por outro lado, não podemos esquecer que essa é uma categoria ideal. Assim, a maioria das empresas que passam por processos de virtualização está em estados intermediários; ou seja, localizam-se, quanto ao nosso terceiro eixo, em posições de tensão entre os extremos.

Finalmente, é interessante estabelecer uma 
nova analogia, entre a discussão realizada quanto à identidade individual e as mudanças observadas quanto à identidade organizacional. Provavelmente, as organizações, tais como os indivíduos, necessitem de algo como a identidade, não por ser-lhes esta uma característica inerente ou natural, mas porque o mercado exige a expressão de uma. Portanto, tanto no plano organizacional como no individual, a questão talvez seja mais de imagem, estilo e retórica, do que de valores, princípios e características centrais. ${ }^{50}$

\section{COMENTÁRIOS FINAIS}

O presente trabalho partiu do interesse dos autores pelo tema da identidade organizacional e pela sua aplicação na análise das organizações, em especial na compreensão de processos de mudança organizacional.

Inicialmente, vimos como o conceito de identidade remonta às origens da lógica e da filosofia clássica. Vimos também como, a partir dessa origem, o conceito foi fragmentado em diferentes correntes em diferentes campos do conhecimento, cada um tomando o conceito à sua conveniência.

Para mapear esse terreno de alta complexidade e viabilizar o uso do conceito de identidade na análise de fenômenos organizacionais, propusemos um quadro conceitual, sugerindo que o conceito de identidade deve ser entendido à luz de duas dimensões: uma dimensão do objeto focal (identidade de quem?) e uma dimensão da observação (identidade observada a partir do quê?). Mostramos, nesse primeiro quadro conceitual, algumas das contribuições mais significativas sobre o tema, explorando suas interligações.

Em seguida, acrescentamos ao quadro conceitual uma nova dimensão, a da definição de identidade. A partir dessa dimensão, que questiona a próprio conceito de identidade, pudemos discutir a centralidade, a dis- tintividade e a durabilidade da identidade individual e da identidade organizacional.

$O$ trajeto realizado mostra que o conceito de identidade, entendido de forma complexa e integrada, é fundamental para a compreensão de fenômenos organizacionais. Pelo fato de transitar por diferentes níveis e dimensões de análise, ele proporciona novas perspectivas ao entendimento de questões não totalmente esclarecidas por abordagens como cultura, clima, comportamento e mudança organizacional.

\section{Caminhos para futuras pesquisas}

Conforme mencionado, apesar da vasta literatura sobre o tema identidade, são raras as abordagens integradoras. Dentro de um domínio inexplorado, os caminhos para pesquisa estão abertos ao teórico interessado. Entre os vários desdobramentos do presente estudo, consideramos os seguintes especialmente interessantes e atrativos:

- explorar as lacunas - conceitos ainda inexplorados de identidade - apontadas no quadro conceitual, visando torná-lo mais útil para o estudo dos fenômenos organizacionais;

- investigar processos de mudança organizacional, como o da RJ\&M, utilizando o quadro conceitual desenvolvido como recurso analítico e explorando em profundidade a interação entre os diversos níveis de identidade; e

- investigar o processo de construção coletiva da identidade individual e da identidade organizacional, a partir do quadro conceitual expandido com a terceira dimensão.

Nossa expectativa é de que o presente trabalho estimule teóricos e pesquisadores das organizações a aprofundar conhecimentos sobre o tema da identidade na análise das organizações.
50. ALVESSON, M. Op, cit. 